\title{
Introduction to the special issue on Social Media and Government
}

\author{
Rodrigo Sandoval-Almazan ${ }^{\mathrm{a}, *}$, Andrea Kavanaugh ${ }^{\mathrm{b}}$ and J. Ignacio Criado ${ }^{\mathrm{c}}$ \\ ${ }^{a}$ Political and Social Sciences Faculty, Autonomous University of the State of Mexico, Toluca, Estado de \\ México, México \\ ${ }^{\mathrm{b}}$ Center for Human-Computer Interaction, Virginia Tech, Blacksburg, Virginia, USA \\ ${ }^{\mathrm{c}}$ Department of Political Science and International Relations, Innovation, Technology and Public \\ Management Lab, Universidad Autónoma de Madrid, Madrid, Spain
}

\section{Introduction}

Social Media (SM) have become a double-edged sword for governments in recent years. They can be a fast and reliable channel to share information, including emergency communication. At other times, SM can create severe problems. For example, the spread of fake news or conspiracy theories (e.g., related to COVID-19 and the vaccine) has gained momentum through various kinds of SM. Therefore, in an age of accelerating digital change, the study of SM and its relationship to government deserves attention.

SM use by governments started as a new set of communication channels for officials looking for easy, accessible, and cheap media to share news and information about services with constituents, citizens, agency counterparts, and other service users. In the early days, government's SM channels included Facebook, Twitter, and/or YouTube, among others. Over time, this use has evolved into a more engaging and participatory channel for online interaction between citizens and public managers. It has expanded to many more formats and platforms, for example including WhatsApp, Instagram, TikTok, Virtual and Augmented Reality.

During crises, such as the COVID-19 pandemic, SM use by government officials, politicians, and public managers has increased dramatically in the face of lockdowns and other restrictions limiting face-to-face meetings and gatherings. This may in the future become the norm, not the exception, even as the pandemic subsides. Different SM platforms have emerged that facilitate citizens' collaboration, virtual support chains for social causes, civic-oriented hackathons, and innovative projects in virtual environments.

\footnotetext{
${ }^{*}$ Corresponding author: Rodrigo Sandoval-Almazan, Political and Social Sciences Faculty, Autonomous University of the State of Mexico, Toluca, Estado de México, México. E-mail: rsandovala@uaemex.mx.
} 
Today, governments face new challenges in using SM that go beyond simple efficient and effective two-way communication channels. The nature of the interaction has changed: misinformation and biases circulated through SM threaten the perceived reliability of and trust in government messages (Bhuiyan et al., 2021) This has been especially evident during the global pandemic of COVID-19. It is paramount that democratic governments support and promote the scientific evidence for their policies and take necessary steps to counteract misinformation that works against the public interest. At the same time, privacy concerns relating to the commercial and government profiling of SM communication are growing. While greater understanding has resulted from computational analyses of social interactions on SM, there is also a growing concern that the algorithmization of social interactions may impinge on confidentiality or lead to the suppression of free speech, for example, non-democratic government identification of and action against a critic or a journalist. This evolving situation presents a raft of new threats for governments as they seek to engage citizens and other service users, develop public policy, and accommodate conflicting interests and collective action, all while considering the 'new normal' (Cinelli et al., 2020).

New technological developments, such as advances in Artificial Intelligence (AI), data analytics, and immersive experiences, are emerging to help understand SM content, including critical thinking about misleading messages and misinformation. New types of applications and platforms, such as WhatsApp, Telegram, Instagram, TikTok, Snapchat, Linked In, and Virtual and Augmented Reality (VR/AR), can appeal to different users and offer different frameworks for expression and interaction. The various SM messages and interactions lend themselves to computational analysis through opinion mining, content analysis, and sentiment analysis, which can be used to help identify patterns or trends in user communication behavior and effects.

The use and impact of current and emerging SM on government and democratic processes are among the challenges addressed in this particular issue. It features a selection of articles from an Open Call for papers for this journal and the Government and Social Media Track of the 20th and 21st Annual International Conferences on Digital Government Research (dg.o 2019 and dg.o 2020). The countries studied in this special issue - Switzerland, Turkey, Tunisia, the US, Mexico, Netherlands, South Africa, and Brazil, represent diverse regions, including North America, Europe, the Middle East, and Africa.

Our first group of articles focuses on the adoption and models of SM use by governments. In particular, these contributions address the organizational side of SM use by the public sector, focusing on the process of adoption and institutionalization of these technologies at the municipal and local levels of government.

In "Digital communication in Swiss cities: A closer look at the low adoption and usage of social media platforms in a technologically advanced and innovative country," Mabillard, Zumofen, and Keuffer investigate 146 cities with over 10,000 inhabitants in Switzerland to understand SM communication. They find that public managers follow a 'conservative approach' to SM as part of the Swiss organizational culture instead of promoting more citizens' engagement. Their contribution is to dampen the expectation of SM platforms and the digitization of government, caused by the lack of political will and strong resistance to $\mathrm{SM}$ adoption.

The study by Villodre, Criado, Meijer, and Liarte, titled "Organizational models for social media institutionalization: an empirical analysis of Dutch local governments," explores two models of SM institutionalization. This study is based on two stages of analysis: surveys and interviews. The first stage analyzes the most significant city councils in the Netherlands, identifying advanced practices of SM implementation based on a survey to public officials managing SM departments. The second stage employs semi-structured interviews to study the organizational features of two city councils with the highest level of SM institutionalization (Utrecht and Eindhoven). The key finding is the identification of two different organizational models of SM institutionalization. They represent the most advanced stage 
of SM implementation that public organizations can be expected to achieve using the different paths identified. At the same time, this study discovers key variables promoting the formalization of SM in government agencies, including coordination, the use of guidance and protocols, evaluation mechanisms, training, and political support.

The second group of articles focuses on the use of SM platforms by the public sector and critical issues evident in their deployment. Key concerns include issues relating to public value and methodological issues related to content and sentiment analysis.

In their article, "Analyzing social media messages of public sector organizations utilizing sentiment analysis and topic modeling" Yaqub, Chun, Atluri and Vaidya report on sentiment analysis of over 14,000 Facebook messages and almost 29,000 Twitter messages from nine public organizations in the U.S. related to law enforcement, public transport, utility services, and the department of motor vehicles. The authors find an overall negative sentiment in these messages. Other findings include a high frequency of Twitter messages by transit agencies and a low use by law enforcement agencies.

In a case study of Turkey, authors Calhan, Karkin, Hasiloglu, and Ozgul investigate public values in "The reflection of public values in Twitter use of metropolitan municipalities: A content analysis employing the analytic hierarchy process". This study focuses on three public values: transparency, participation, and collaboration. The authors find that metropolitan municipalities in Turkey are not aware of the importance of public values and are not successfully integrating them into their use of SM. In discounting the public value of civic participation, for example, the government does not reply to all citizens' messages. Evidence that transparency is undervalued includes the use of incomplete information, mainly budgetary information. They propose prospective studies to identify how local governments evaluate participation, collaboration, and transparency in their operations.

In "Correlations of social media performance and electoral results in Brazilian presidential elections", Brito, Meira, and Adeodato present a content analysis of Facebook, Instagram, and Twitter posts concerning the outcome of presidential elections in Brazil. The authors use content analysis to identify relevant, even contentious, topics and correlation analysis to test for possible connections between posting activity and election results. Interactions around posts were the highest on Instagram, where there was increased attention given to political content. The authors propose a new set of metrics for modeling SM performance and report significant correlations between SM activity and electoral performance.

The third group of articles focuses on the use of SM by governments during a crisis or emergency. The utilization of SM to manage crisis situations is well-established. This group of articles contributes case studies using attribution theory and mixed methods to analyze the government's use of SM during a water crisis and the COVID-19 pandemic.

SM use by a variety of officials and other decision-makers is increasing in many agencies. In the case study entitled "The use of social media for public engagement during the water crisis in Cape Town", Gwaka and Waxa analyze the use of Twitter by the government of Cape Town, South Africa during the water crisis of 2016-2018. Using attribution theory and a mixed-methods approach (with interviews, ethnography, and survey questionnaires), the authors study the combination of affordances (i.e., properties enabling certain uses) of SM technologies and the actors' interpretation of events. The study confirms that local public authorities used SM for intensely engaging with citizens during the crisis. Notably, the meta-voicing affordance facilitated co-construction of the story during the crisis, and visibility paved the way for previously unusual debates and participation with the public. At the same time, the emerging narratives during the water crisis mirrored structural elements of the local society (i.e., corruption or segregation). In using attribution theory they identify and contribute to understanding actors' strategies in SM use during such events. 
A complementary perspective is given in an article by Sandoval-Almazan and Valle-Cruz entitled "Social media use in government health agencies: the COVID-19 impact". This longitudinal study from 2017-2020 compares SM communications within a local government agency of the central State of Mexico, before and after the COVID-19 pandemic. The findings note that tweets and Facebook posts are an efficient means of communication during a crisis. They also help explain how external factors determine the use of different SM platforms. Here, the number of interactions is related to the internal organization of the agency, and influences the rate of growth of followers on Twitter and Facebook. The authors conclude that government agencies underestimate SM platforms for their communication because they do not have a strategy or promote bidirectional communication with citizens.

The last group of articles relates to the use of SM by citizens for political participation. This approach to political participation is broad in scope as SM platforms have opened up new and different digital opportunities to expand political participation, including e-petitioning, virtual reality, or the use of different media to access political information under different political conditions.

Dumas investigates extremism in "Electronic petitioning as online collective action: Examining the e-petitioning behavior of an extremist group in We the people." She presents a case study of user behavior toward online petitioning, studying the first online US government petitioning system established under the Obama administration. She uses data mining (social network analysis and 'market basket' analysis) to analyze data from 125 petitions created by a white supremacist group. The author finds evidence of social mobilization advocating white supremacy through the platform.

In "Virtual Reality-Driven Serious Communication: Through VR-Dialogue towards VR-Participation" Porwol and Ojo explore the potential of citizens' political participation via Virtual Reality (VR). The authors discuss the interplay between e-participation and emerging social VR technologies, analyze their affordances, and evaluate principles of dialogue (listening, respecting, suspending, and voicing). The authors build a framework for VR-mediated communication that connects general dialogue principles with immersive VR principles (immersion, presence, and sense of community). This opens a window to new spaces and arenas for social VR.

In "Media Use, Information Reliability and Political Efficacy in Tunisia, 2011-2019" Kavanaugh, Sheetz, Skandrani, and Sghaier examine changes over time in the use by young, educated Tunisians of different mediated political information sources and their effect on political information efficacy (sense of being well-informed). Guided by communication behavior and political participation theories, the article reports results from the most recent of three surveys administered to different, but comparable opportunity samples of young, educated Tunisians, regarding the media they used to obtain political information during the 2011 Arab Spring uprising, the 2014 elections, and the 2019 elections. The results confirm exploratory findings that show increases in perceived reliability of government information sources during the 2014 and 2019 elections and decreases in perceived reliability of SM since the 2011 revolution. Results also confirm that higher perceptions of information reliability and information sharing lead to greater political information efficacy, an established predictor of further democratic participation.

\section{Implications for research and practice}

There are important implications for research and practice that arise from the articles in this special issue.

With regard to the adoption of SM by government, we learn in the case study of Swiss cities by Mabillard and colleagues that some communication managers do not see SM as an essential tool for communication and do not regard these platforms as adding any value to their existing forms of communication. Most 
cities using SM platforms still lack a comprehensive strategy, they lack a global approach to SM adoption and use and lack the political will to promote SM use in their cities. The authors conclude that SM adoption in public administrations of Switzerland should be analyzed under the prism of risk to ensure a coherent mitigation strategy.

For the institutionalization of SM use, government agencies should consider the key variables identified by Villodre and colleagues, including: coordination; the use of guidance and protocols; evaluation mechanisms; training; and political support. This research also confirms the idea of institutionalization as a process with different paths to fulfillment. So, in order to have a high level of SM institutionalization, public organizations should have diverse SM management strategies. At least two different models of institutionalization exist (centralized or distributed), with differentiated types of coordination.

In the topic area of SM use by the public sector, new challenges arise around government collaboration, the diffusion of public values, and greater transparency in elections. For example, based on their case study of municipalities in Turkey, Chauhan and colleagues recommend that public officials conduct studies to identify how local governments evaluate participation, collaboration and transparency in their operations. Many local governments are not fully aware of the significance of public values and thus are not so successful in reflecting public values in their communications, particularly when using SM. They recommend that governments pay attention to the motivations of local stakeholders when they interact with their local governments. In this respect, policymakers need to continuously update SM policies and guidelines.

During the pandemic, general lockdowns and other restrictions on rallies and gatherings made online campaigning one of the main modes for candidate contact and interaction with voters. It is anticipated that the use of online formats and SM by candidates will continue in the post-pandemic era. Metrics such as those proposed by Brito, Meira, and Adeodato to evaluate the use and impact of SM on election outcomes will inevitably become more important. As they note, in the electoral setting, new datasets are likely to be used, such as campaign pools and demographic data. These data, along with AI techniques, such as natural language processing and sentiment analysis are powerful tools for future electoral forecasting.

The topic of SM use by governments during crises and emergencies contributes several critical points for both research and practice. Here, the paper of Gwaka \& Waxa raises the issue of how SM platforms are used during the crisis and the interpretation that different actors make of these uses. In particular, it is noted how governments may use SM for many other purposes (in the case of the City of Cape Town water crisis, metavoicing, persistent engagement, and visibility), while at the same time managing their relations with citizens. These interaction processes can make a difference regarding the outcomes of the use of SM during a crisis situation. Public officials should work very closely with societal conditions 'on the ground' to avoid dissonant messages that might undermine citizens' confidence during moments of crisis.

Sandoval-Almazan and Valle-Cruz's article contributes to our understanding of public health communication using SM via its focus on public managers. Much prior research focuses on citizen's use of SM during crises (Starbird et al., 2010; Soden and Palen, 2018). This paper contributes to our understanding of the communications perspective of government during crisis (Kavanaugh, et al., 2012, among others) Governments can reach diverse users (whether internal or external to the administration) by using different platforms to send the same or sometimes different messages, depending on each platform's affordances and types of users. Increasing followers on multiple platforms can help governments reach the broadest and most diverse populations with urgent information and feedback.

The topic of citizen's use of SM for political participation has a long history, with implications for civic engagement as well as misinformation. It raises concerns about interaction, veracity and reliability, 
privacy, and democratic debate. Citizen use of SM can bring to the government useful insights and ideas, as well as harsh criticism and false claims. A platform for online petitioning can be helpful for the government to receive citizen suggestions for policymaking. However, as we learned in the study by Dumas, such platforms can also help organize and mobilize groups with policies and goals that are exclusionary, sometimes violent, and against other citizens and the public interest. Governments with e-petitioning platforms should be aware of important information that petition data can provide when it is collected and analyzed.

Porwol and Ojo have indicated that emerging platforms, such as Virtual Reality (VR) and its social affordances (e.g., presence, interaction) offer opportunities for citizens to engage with each other and with policy issues that are better understood and shared in an immersive or 3D environment. These include spatial impacts on land use from downtown sprawl or commercial development and other long-term planning that benefits from 3D data visualization or immersion. By offering multiple modes (in-person, print, online, 2D, 3D) in which citizens can engage in data sharing, interaction, and public discussion, there is a greater probability that civic participation will be broader and more diverse.

In examining different media for different sources of political information, the Internet and SM can be the most reliable and trusted sources of political information under conditions of authoritarian regimes or revolution, as shown in a longitudinal study of Tunisia by Kavanaugh, Sheetz, Skandrani and Sghaier. Post-revolution, however, as more liberal government coalitions emerged easing press restrictions and censorship, SM became a less reliable source of political information than official sources (whether broadcast TV or government websites). Greater freedom of the press under more democratic postrevolution governments leads to perceptions of increased reliability of these information sources, and a higher sense of being well-informed (i.e., political information efficacy). These outcomes are known to lead to greater political participation, thus suggesting a strengthening and consolidation of democratic practices.

Looking ahead, many of the research issues and implications discussed in this Special Issue will persist, even as the technologies and their affordances evolve. We have seen new platforms emerge, such as TikTok, WhatsApp, and other SM. Micro-blogs, such as Twitter, and social network sites, such as Facebook, are well established and reliable, but they may be less innovative than emerging applications. Tik Tok, Instagram, Telegram, Zoom, and What's App have helped improve, create and change the way we have been interacting. The TikTok 'revolution' rejuvenates the SM sphere in many fields, not just in digital government. In the digital government domain alone, many changes are evident. The new privacy rules of Facebook, Twitter, and more recently, Apple and Google have changed relations among users and companies, creating friction and distrust on both sides. Also, governments worldwide are forced to mediate in this conflict and develop rules to protect users' privacy. This Special Issue seeks to highlight some of the state-of-the-art in SM use in governmental settings and glimpse some of the near future challenges and offerings.

The practice of SM management and use by government needs to be developed in order to fulfill the potential promises of these technologies. Departments and units specializing in managing SM play a critical role in fostering different capabilities, and beyond the simple communication function. Promoting transparency accessing public information, enhancing citizens' participation are great examples of advanced uses of SM in government settings. Practitioners should also encourage citizen interaction with public institutions to help counteract misinformation and polarization of political discourse. Governments should target a more diverse demographic profile of employees working as SM strategists and managers, including people from underrepresented groups and others with traditionally limited engagement with government. SM use could help practitioners surpass the intermediation crisis of governments and public administrations with more pluralistic approaches which may in turn bolster trust in public institutions. 
Over the past two decades, starting with the earliest government Internet Relay Chats, discussion forums, and weblogs, such as those in the Town of Blacksburg, Virginia (Martin \& Cohill, 2000; Kavanaugh et al., 2010) among many other examples, SM has expanded the interactive channels of governments and public organizations, and created new ways of reaching out to service users and citizens. These processes will probably evolve and transform soon in ways we don't even imagine. The diversity of platforms, audiences, uses, metrics, and contexts will challenge the research agendas of the international scholarly community studying these developments. We hope to see more comparative studies and scholarly dialogue to better understand their use and impacts. Shared knowledge of best practice and experience help public officials and managers to expand their managerial toolkit and to work with SM technologies more effectively. With this Special Issue, we seek to contribute to emerging research agendas, as well as knowledge that will inform practical implications of SM in government.

\section{Acknowledgments}

We would like to thank the authors for their contributions, patience, and insights into this fast changing field of research. In addition, the expertise and insightful comments provided by reviewers have been very valuable in improving the manuscripts. We thank them for their generosity. We also want to thank everyone involved in making this Special Issue possible at Information Polity, in particular, Albert Meijer and William Webster, the co-editors in chief, for encouraging and supporting us, and for their valuable feedback as the Special Issue was taking shape. Finally, we are grateful to Gabriela Ricci, for effectively managing all the manuscript production process.

\section{References}

Bhuiyan, M. M., Horning, M., Lee, S. W., \& Mitra, T. (2021). NudgeCred: Supporting News Credibility Assessment on Social Media Through Nudges. Proceedings of the ACM on Human-Computer Interaction, Volume 5, Number CSCW2, Article 427 (October 2021), 30 pages. doi: 10.1145/3479571.

Cinelli, M., Quattrociocchi, W., Galeazzi, A., Valensise, C. M., Brugnoli, E., Schmidt, A. L., .. \& \& Scala, A. (2020). The COVID-19 social media infodemic. Scientific Reports, 10, 1-10.

Martin, C., \& Cohill, A. 2000 (revised edition). Managing Information in a Community Network, 281-312. In A. Cohill and A. Kavanaugh (Eds.) Community Networks: Lessons from Blacksburg, Virginia. Norwood, MA: Artech House.

Kavanaugh, A., Fox, E., Sheetz, S., Yang, S., Li, L.T., Shoemaker, D., Natsev, A., \& Xie, L. (2012). Social Media Use by Government: From the routine to the critical. Special Issue on Social Media in Government. Government Information Quarterly, 29(4): 480-491.

Kavanaugh, A., Pérez-Quiñones, M., Tedesco, J., \& Sanders, W. (2010). Towards a Virtual Town Square in the Era of Web 2.0, In J. Hunsinger, L. Klastrup and M. Allen (Eds.) Handbook of Internet Research, NY: Springer, pp. 279-294.

Soden, R., \& Palen, L. (2018). Informating Crisis: Expanding Critical Perspectives in Crisis Informatics. In Proceedings of the ACM on Human-Computer Interaction Volume 2, Issue CSCW November 2018, Article No.: 162, pp. 1-22, doi: $10.1145 / 3274431$.

Starbird, K., Palen, L., Hughes, A. L., Vieweg, S. (2010). Chatter on the red: what hazards threat reveals about the social life of microblogged information. In Proceedings of the 2010 ACM Conference on Computer Supported Cooperative Work (CSCW 2010): 241-250. 